\title{
Cellular Immune Responses and Immune Escape Mechanisms in Breast Cancer: Determinants of Immunotherapy
}

\author{
Christoph Domschke ${ }^{a} \quad$ Andreas Schneeweiss $^{a}$ Stefan Stefanovic $^{\text {a }}$ Markus Wallwiener $^{\text {a }}$ \\ Joerg Heil ${ }^{a}$ Joachim Rom ${ }^{a}$ Christof Sohn $^{a}$ Philipp Beckhove ${ }^{b}$ Florian Schuetz $^{a}$ \\ a Department of Gynecology and Obstetrics, Heidelberg University Hospital, National Center for Tumor Diseases (NCT), Heidelberg, Germany \\ ${ }^{\mathrm{b}}$ Regensburg Center for Interventional Immunology (RCI) and University Medical Center of Regensburg, Regensburg, Germany
}

\section{Keywords}

Breast cancer - Cellular immunity · Immune escape . Immunoediting · Immunosurveillance $\cdot$ Immunotherapy

\section{Summary}

More recently, immunotherapy has emerged as a novel potentially effective therapeutic option also for solid malignancies such as breast cancer (BC). Relevant approaches, however, are determined by the 2 main elements of cancer immunoediting - the elimination of nascent transformed cells by immunosurveillance on the one hand and tumor immune escape on the other hand. Correspondingly, we here review the role of the various cellular immune players within the host-protective system and dissect the mechanisms of immune evasion leading to tumor progression. If the immune balance of disseminated BC cell dormancy (equilibrium phase) is lost, distant metastatic relapse may occur. The relevant cellular antitumor responses and translational immunotherapeutic options will also be discussed in terms of clinical benefit and future directions in BC management.

(C) 2016 S. Karger GmbH, Freiburg

\section{Introduction}

An intact immune system plays a dual role in cancer: it can prevent/control as well as shape/promote cancer by a process called 'cancer immunoediting' [1]. On the one hand, in a host-protective elimination phase named 'immunosurveillance', the immune system can recognize and suppress tumor growth by destroying nas- cent transformed cells or inhibiting their outgrowth. This antitumor immunity is substantiated by the main cellular effectors of the innate and the adaptive immune system, namely natural killer cells, natural killer T cells, and T cells (TCs), as well as increased proimmune humoral factors (e.g., interferons) in the tumor microenvironment. On the other hand, in the tumor-promoting phase referred to as 'immune escape', the immune system can further tumor progression either by selecting cancer cells that are more capable of surviving the host's immunocompetence or by modifying the tumor microenvironment in such a way that tumor outgrowth is facilitated [2]. In between the above phases is the equilibrium where cancerous cells are kept under control but are not eliminated by the immune system. This balance of antitumor and tumor-promoting factors may maintain the tumor in a functionally inactive state of dormancy over a period of many years [3].

The processes mentioned above also make up the rationale for the development of immunotherapeutic options in breast cancer (BC) [3-5], as characteristically in this tumor entity, already at very early stages, cancer cells are able to disseminate hematogenously from the primary tumor site, and distant metastases often occur only after many years of latency [6]. In this context, one predominant organ associated with the dissemination and survival of $\mathrm{BC}$ tumor cells is, besides others such as locoregional lymph nodes, the bone marrow (BM). Of note, the detection of disseminated BM tumor cells correlates with an increased rate of secondary osseous and visceral metastases and with a worse overall survival [7-11].

Consequently, in addition to surgical resection of the primary tumor and locoregional irradiation, curative BC therapy aims at eliminating disseminated micrometastatic tumor cells. In this context, besides cytostatic and/or hormonal therapies, new supportive treatment options like immunotherapy are increasingly gaining oncological interest. Hence, we also review aspects of $\mathrm{BC}$ immunoediting processes with respect to potential immunotherapeutic approaches.

\section{KARGER \\ Fax +497614520714

() 2016 S. Karger GmbH, Freiburg

$1661-3791 / 16 / 0112-0102 \$ 39.50 / 0$
PD Dr. Christoph Domschke 


\section{Cellular Immune Responses}

\section{Tumor Site}

In human BCs, like in other malignancies, the presence of primary tumor-infiltrating lymphocytes (TILs), especially of the Th1 and cytotoxic variety, is correlated with the absence of metastatic invasion and improved clinical outcome in terms of overall survival rates [12]. This holds particularly true for BCs with aggressive features such as high histologic grade or estrogen receptor- $\alpha$ negativity $[13,14]$.

Intriguingly, in triple-negative invasive $\mathrm{BC}$ patients receiving neoadjuvant chemotherapy and subsequent surgical treatment, the immunohistochemical or mainly the hematoxylin and eosin staining analysis of primary tumor needle biopsy specimens revealed that the pathologic complete response rates of tumors showing a high TIL score were significantly higher than those of tumors with a low TIL count $[15,16]$. These results suggested that the pretreatment host immune response may enhance the ability of anthracycline/taxane-based neoadjuvant chemotherapy to eliminate cancer cells [17]. This hypothesis was further corroborated by large studies which demonstrated triple-negative as well as HER2-overexpressing BC phenotypes with high levels of intratumoral cytotoxic TCs to have heightened sensitivity to anthracycline-based chemotherapy, as assessed by the immediate response to neoadjuvant therapy and long-term disease-free survival rates [18-20]. Further, in HER2-positive BC patients, high levels of TILs were also found to be associated with improved therapeutic responses to the monoclonal antibody trastuzumab [21].

In summary, the data available suggest that particularly in aggressive subtypes such as triple-negative and HER2-positive BC the immune response plays a pivotal part in tumor chemosensitivity and clinical outcome.

\section{Bone Marrow Site}

TCs play a central role in cell-mediated tumor immunity. Conventionally, the precursors of TCs are produced in the BM but subsequently leave the BM and mature in the thymus. Mature cells then emigrate from the thymus into the circulation. Repeatedly, circulating naive TCs leave the blood to enter peripheral lymphoid organs where they may encounter their specific tumor antigen and become activated as effector (cytotoxic or helper) TCs.

Relating to BC immunity and the generation of tumor-specific effector and memory TCs, blood/BM interactions proceed in 3 steps $[22,23]$ :

i) Naive TCs as well as tumor cells are recruited from the circulation to the BM ('homing') via constitutively expressed adhesion molecules [24, 25];

ii) BM-resident antigen-presenting cells, particularly dendritic cells, can scan, process, and cross-present BC-associated antigens to prime TCs $[22,26]$; and

iii) activated TCs proliferate and may become effector and memory cells that either recirculate or remain in the BM compartment.

The coexistence of BM-resident tumor-specific TCs and disseminated $\mathrm{BC}$ cells maintains the quiescent state, i.e., the immune balance, which is referred to as tumor dormancy, while a loss of TC function can lead to tumor metastasis even after years of latency [27-30].

Furthermore, especially in the case of BC, in a significant number of patients during the course of disease, tumor-specific TC responses could be proven to have been induced and maintained in the form of BM memory TCs (TMCs) - a subset of BC-specific TCs that persist long-term [31].

TMCs are an ideal source for the generation of therapeutic effector TCs expressing the CD8 glycoprotein at their surfaces. This is due to the fact that secondary CD8+ TC responses take place more quickly and more effectively than primary responses [32-34]. Correspondingly, in a trial of advanced metastasized BC patients with tumor-reactive TMCs in the BM, no tumor-specific TCs were detected in the peripheral blood at the beginning. After therapy with reactivated autologous BC-reactive BM TMCs, however, about $44 \%$ of patients showed tumor-specific TCs in the peripheral blood $[35,36]$.

\section{Tumor Immune Escape}

\section{Mechanisms}

In the studies cited above $[35,36]$, which will be discussed in more detail in the subsequent chapters, roughly half of the BC patients treated did not respond to the immunotherapy employed. In these patients, counterregulation mechanisms may have taken action leading to post-therapy tumor immune escape along the following lines:

i) The immune recognition of tumor cells can be circumvented because of loss of tumor antigen expression that may occur in at least 3 ways [2]: through development of tumor cells lacking expression of potent rejection antigens; by means of downregulation of major histocompatibility complex (MHC) class I proteins that present these antigens to tumor-specific TCs; or via loss of tumor cells' antigen processing capacity that is mandatory to develop the antigenic peptide epitopes and load them onto the MHC class I molecules.

ii) At the tumor cell level, resistance to cytotoxic lysis by immune cells may be brought about by enhanced expression of antiapoptotic effector molecules such as FLIP and BCL-XL [37] or by persistent activation of pro-oncogenic transcription factors like STAT3 [2]. Alternatively, tumor cells can evade immunemediated killing through expression of mutated inactive forms of death receptors [37]. Concertedly, these mechanisms may promote tumor progression.

iii) Tumor immune escape may develop due to local immunosuppression in the tumor microenvironment. Such an immunosuppressive state can be established either by tumor cells producing immunosuppressive cytokines (e.g., vascular endothelial growth factor and transforming growth factor- $\beta$ (TGF- $ß$ )) or by recruitment of specific immunosuppressive leukocyte populations (e.g., regulatory TCs (Tregs) and myeloid-derived suppressor cells) $[2,37,38]$. 
Clearly, the rate of $\mathrm{BC}$ progression is determined by the balance between the above immune-inhibitory factors of tumor immune escape and the host-protective capacity of the immune system as well as the immune-stimulatory conditions of immunotherapeutic approaches.

\section{Potential Anti-Escape Strategies}

In BC patients with failed immune response to cellular immunotherapeutic approaches, a significantly higher proportion of Tregs was found in the BM [35]. Tregs are a subpopulation of TCs which generally suppress (hence also called suppressor TCs) or downregulate activities of effector TCs thus modulating immune reactions, maintaining tolerance to self-antigens, and abrogating autoimmune disease. Consequently, in future immunotherapy studies, patients might benefit from ex vivo Treg depletion prior to TC stimulation.

Additional strategies potentially capable of reducing the immunosuppressive effects of Tregs are currently investigated in murine models or first clinical studies and comprise i) denileukin diftitox, a fusion protein of interleukin 2 and diphtheria toxin targeting Treg cells [39-41], ii) direct antibody blockade of the immunosuppressive moieties of Tregs [37, 38], and iii) pharmacological agents such as cyclooxygenase-2 inhibitors as well as the antineoplastics temozolomide (tumor DNA methylator), fludarabine (purine analog), and cyclophosphamide (CTX) [42, 43]. Strikingly, CTX, a well-known chemotherapeutic compound, when being applied solely at low doses in a metronomic regimen could be demonstrated to induce a selective profound reduction in circulating Tregs through enhanced apoptosis and decreased proliferation of this cell type $[44,45]$. Simultaneously, spontaneous antitumor TC responses were found restored $[42,45]$.

Correspondingly, in a recent study of metastasized BC patients, Ge et al. [46] reported metronomic CTX treatment over 3 months to cause a transient reduction in circulating Tregs by more than $40 \%$ associated with a strong and lasting increase in breast tumor-specific TCs, which significantly correlated with disease stabilization and overall survival. Consequently, low-dose CTX might be successfully integrated into a future concept of chemoimmunotherapy.

\section{Cellular Immunotherapy}

\section{Possible Accesses}

Cellular immunotherapy approaches are based on 2 different principles [47]:

i) The body's own immune system can be actively and specifically stimulated through confrontation of immune cells with autologous or allogeneic tumor antigens in situ. This leads to a primary activation of naive effector cells or to a secondary reactivation of memory cells which were formed during an earlier confrontation of the immune system with the specific antigen. This procedure corresponds to an active immunization, i.e., a vaccination.

ii) Autologous or allogeneic immune cells (tumor-specific TMCs from BM [35, 36] or peripheral blood [48], TILs [49], or engi-

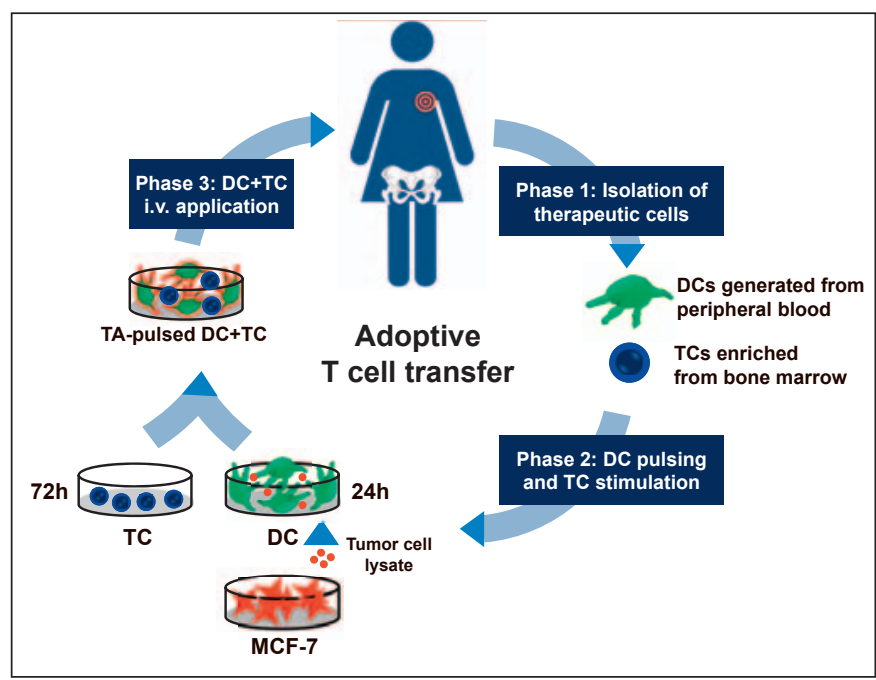

Fig. 1. Adoptive immunotherapy (ADI) of metastasized breast cancer patients. Process of ADI treatment preparation $(\mathrm{PB}=$ Peripheral blood; $\mathrm{BM}=$ bone marrow; $\mathrm{DC}=$ dendritic cell; $\mathrm{TC}=\mathrm{T}$ cell; $\mathrm{MCF}-7=$ human breast cancer cell line MCF-7; TA = tumor antigen). Adapted from [36, 57].

neered TCs [50]) with a specific affinity for tumor-associated antigens can get activated ex vivo and subsequently applied directly into the human organism as cellular immunotherapy. This is equivalent to a passive immunization, i.e., an adoptive immunotherapy (ADI).

\section{Vaccination}

Vaccine-based therapies do firstly require the identification of tumor-specific antigens (expressed on malignancies only) and tumor-associated antigens (expressed on all types of cells but overexpressed on cancer cells). In breast cancer, relevant antigens that could be targeted by vaccination include MUC-1, MAGE-A3, and NY-ESO-1 which are characteristically expressed on estrogen receptor-negative and/or triple-negative tumor subvariants $[51,52]$. In vaccination strategies, liposomebased and synthetic peptide vaccines are employed. Additionally, the administration of in vitro activated dendritic cells can be involved [53]. In BC patients, attempts along the above lines are currently being evaluated in clinical trials which are comprehensively expounded elsewhere [54].

\section{Adoptive Immunotherapy}

Results from animal experiments [55-57] were the rationale for the establishment of a phase I trial where patients with advanced metastasized $\mathrm{BC}$ were treated with reactivated autologous tumorspecific BM TMCs $[35,36]$. In $16 \mathrm{BC}$ patients with tumor-reactive BM TMCs, another BM aspiration was performed which provided tumor-specific TMCs for the following flow of ADI treatment:

i) TMCs were activated by antigen-pulsed dendritic cells with antigens originating from lysates of a microbiologically tested MCF-7 cell line. After incubation of the antigen-presenting cells with TMCs, the cell suspension $\left(2 \times 10^{6}\right.$ to $\left.5.7 \times 10^{7} \mathrm{TCs}\right)$ was intravenously applied under antibiotic prophylaxis (fig. 1). This 
immune cell transfer was well tolerated except for influenza-like symptoms in 2 patients.

ii) As early as 1 week after intravenous ADI, tumor antigen-reactive TMCs could be detected in the peripheral blood of about $50 \%$ of the patients (ADI responders). The responding patients had received the highest total number of TMCs and harbored the lowest tumor burden. Of note, significantly higher levels of tumor-specific TMCs had been observed in the BM of patients with subsequently positive ADI response compared to nonresponding patients.

iii) In none of the treated patients with overt bone metastases tumor-reactive TCs were detectable in the peripheral blood after ADI. This massively reduced immune responsiveness may be related, at least in part, to the increased release of immunosuppressive TGF- $\beta$ from the bone during the osseous metastatic process $[36,58-61]$.

iv) Finally and most importantly, ADI responders had a significantly longer median survival than non-responders (58.6 vs. 13.6 months; $\mathrm{p}=0.009$ ) with 3 out of 16 patients still being alive at last follow-up and more than 7 years after ADI [36].

In summary, results hint at a relationship between immune response and cancer prognosis and suggest that preferably $\mathrm{BC}$ patients without bone metastases but with immunologic response to adoptive TC transfer might benefit from this treatment option.

\section{Perspectives}

The central aim of forthcoming studies will be the control of tumor escape mechanisms as described in the preceding chapters.

Firstly, approaches to deplete or reduce Tregs in their capacity to suppress the immune system might substantially improve spontaneous or immunotherapy-related tumor defense. One strategy in this context may be a combined chemoimmunotherapy of metronomic CTX with an adoptive TC transfer (ADI). This might include a selection of defined tumor antigens with immune adjuvants preventing the re-activation of type- 2 TC responses during stimulation. Additionally, new approaches to polyclonal TC expansion deserve consideration attempting to replace autologous dendritic cells by artificial antigen-presenting cells with improved TC-stimulatory properties [62].

Secondly, although still in the experimental phase, recent initiatives aim at grafting patients' primary TCs with a second TC receptor known to recognize a defined tumor antigen [47]. There is hope that, in the long run, such engineered tumor-specific TCs (CAR-T cells) may be successfully used for adoptive immunotherapy purposes $[63,64]$.

Thirdly, other current attempts focus on adoptive TC therapy with TILs [65-68]. In this context, tumor-reactive TCs are harvested from tumor-infiltrated lymph nodes or tumor tissue. In metastatic melanoma patients, a transfer of ex vivo activated and expanded autologous TILs after a preceding lympho-depleting chemotherapy was demonstrated to induce tumor regression in about $50 \%$ of treated patients $[67,68]$. Therefore, a pivotal scien-

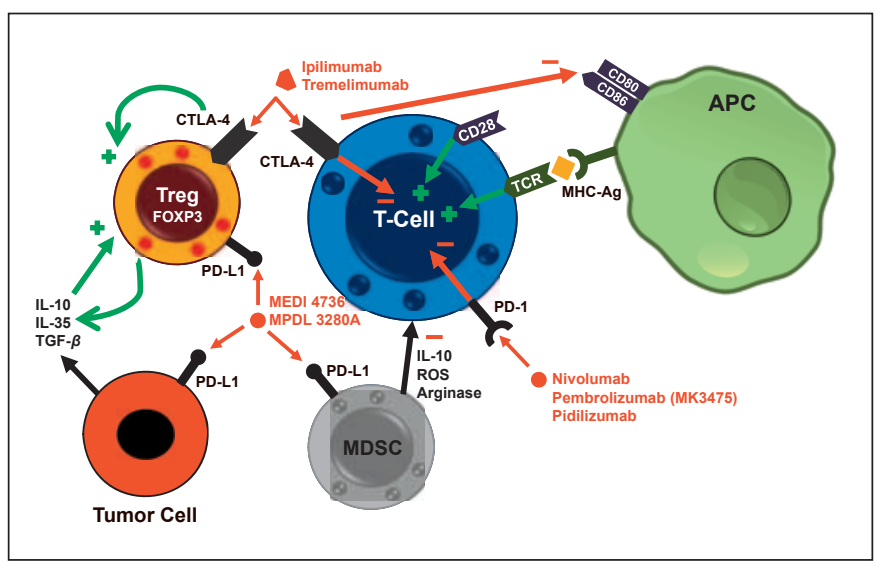

Fig. 2. Checkpoint inhibitors (CTLA- $4=$ Cytotoxic T-lymphocyte-associated antigen 4; PD-(L)1 = programmed cell death protein (ligand) 1 ; APC = antigenpresenting cell; $\mathrm{MDSC}=$ myeloid-derived suppressor cell; $\mathrm{ROS}=$ reactive oxygen species; TCR $=$ T cell receptor; MEDI 4736/MPDL 3280A = anti-PD-L1 monoclonal antibodies). Adapted from [54].

tific focus is on the improvement of the tumor antigen recognition capacity and, hereby, the therapeutic efficacy of TILs or genetically engineered TCs [68-78].

Finally, immune checkpoints are currently the focus of clinical research (fig. 2). The expression of 'cytotoxic T-lymphocyte-associated antigen 4' (CTLA-4) on the plasma cell membrane of TCs induces a downregulation of their activity and thereby leads to immunosuppression. This effect is mediated by B7 expression on antigen-presenting cells as for example dendritic cells. CTLA-4 is in competition with stimulating CD28 for binding to B7. Therefore, the expression of one or the other results in TC suppression (CTLA-4) or stimulation (CD28).

The 'programmed cell death protein-1' (PD-1) is also expressed on the plasma cell membrane of TCs. Activated by its ligands PD-L1 and PD-L2, the generation of Tregs is induced and the activity of immune cells is downregulated [79]. PD-L1 is expressed by about $30 \%$ of BC cells and results in relevant immunosuppression [80].

Several immune checkpoint inhibitors are now under clinical investigation. Nivolumab, pidilizumab, and pembrolizumab are anti-PD-1 antibodies. In metastatic BC, a study with pembrolizumab has just been presented [81]. In only $18.5 \%$ of the patients included a clinical response was detected. Nevertheless, this response was long-lasting. Nivolumab showed a clinical benefit in patients with malignant melanoma where cases expressing PD-L1 on the tumor cells were correlated with best responses [82]. In those patients, even a prolonged overall survival was observed. BCrelated data is promising although systematic studies are still missing.

As described above, blockade of CTLA- 4 by antibodies such as ipilimumab or tremelimumab enhances the immune reaction against tumor cells. In malignant melanoma patients, ipilimumab resulted in favorable clinical responses. Consequently, this antibody is now being tested in other solid carcinomas, with BC-related data still missing. Nevertheless, a combination of checkpoint inhibitors might be a promising therapeutic option. In patients with malig- 
nant melanoma, for instance, ipilimumab together with pembrolizumab was tested against a solitary ipilimumab therapy. This trial showed a relevant benefit in the cohort of patients with combined therapy thus justifying further studies [83]. Modulating the immune system, however, is not free of clinical side effects. Suppressing immunosuppressive effects may be related e.g. to several autoimmune reactions (thyroiditis, colitis, pancreatitis etc.). Therefore, thorough clinical observation within trials is mandatory.

All in all, in contrast to other neoplasms such as melanoma, BC treatment with immune checkpoint-abrogating agents has only recently become a field of interest. Hence, most clinical trials based on immune checkpoint blockade are pilot or phase I/II studies [54].

\section{Conclusion}

In $\mathrm{BC}$, the presence of TILs has been shown to correlate with a favorable long-term prognosis primarily of high-grade/highly proliferative lesions. TILs were also associated with a favorable response to neoadjuvant and adjuvant anthracycline-based chemotherapies. These findings support the addition of immunotherapeutic strategies to conventional treatment concepts.
In late-stage metastasized $\mathrm{BC}$ patients, the adoptive transfer of BM TCs (ADI) can induce the presence of tumor antigen-reactive TCs in the peripheral blood. This positive immunologic response appears to depend significantly on the number of transferred specific memory TCs and on the absence of BM metastases. Immune responders show a significantly prolonged overall survival.

Eventually, future strategies to potentially overcome tumor immune escape should comprise Treg depletion from BM preparations before their ex vivo activation. Additionally, a functional inhibition of the immunocompromising capacity of Tregs or a functional TC stimulation may be achieved either by directly targeted antibodies (CTLA-4, PD-1, PD-L1, IL-2 receptor) or by pharmacologic agents such as cyclooxygenase- 2 inhibitors or CTX. Besides a checkpoint inhibition, immunomodulating metronomic low-dose CTX plus ADI appears to be a promising choice of combinatorial chemoimmunotherapy. Finally, current developments relating to artificial antigen-presenting cells, engineered tumor-specific TCs, and TILs should be followed up.

\section{Disclosure Statement}

No conflicts of interest were disclosed.

\section{References}

1 Mittal D, Gubin MM, Schreiber RD, Smyth MJ: New insights into cancer immunoediting and its three component phases - elimination, equilibrium and escape. Curr Opin Immunol 2014;27:16-25.

2 Schreiber RD, Old LJ, Smyth MJ: Cancer immunoediting: integrating immunity's roles in cancer suppression and promotion. Science 2011;331:1565-1570.

3 Dushyanthen S, Beavis PA, Savas P, et al.: Relevance of tumor-infiltrating lymphocytes in breast cancer. BMC Med 2015;13:202.

4 Datta J, Berk E, Cintolo JA, et al.: Rationale for a multimodality strategy to enhance the efficacy of dendritic cell-based cancer immunotherapy. Front Immunol 2015;6:271.

5 Ravelli A, Reuben JM, Lanza F, et al.: Immune-related strategies driving immunotherapy in breast cancer treatment: a real clinical opportunity. Expert Rev Anticancer Ther 2015;15:689-702.

6 Demicheli R: Tumour dormancy: findings and hypotheses from clinical research on breast cancer. Sem Cancer Biol 2001;11:297-306.

7 Diel IJ, Kaufmann M, Costa SD, et al.: Micrometastatic breast cancer cells in bone marrow at primary surgery: prognostic value in comparison with nodal status. $J$ Natl Cancer Inst 1996;88:1652-1658.

8 Braun S, Vogl FD, Naume B, et al.: A pooled analysis of bone marrow micrometastasis in breast cancer. N Engl J Med 2005;353:793-802.

9 Domschke C, Diel IJ, Englert S, et al.: Prognostic value of disseminated tumor cells in the bone marrow of patients with operable primary breast cancer: a long-term follow-up study. Ann Surg Oncol 2013;20:1865-1871.

10 Hartkopf AD, Taran FA, Wallwiener M, et al.: Prognostic relevance of disseminated tumour cells from the bone marrow of early stage breast cancer patients - results from a large single-centre analysis. Eur J Cancer 2014;50:2550-2559.
1 Banys M, Krawczyk N, Fehm T: The role and clinical relevance of disseminated tumor cells in breast cancer. Cancers (Basel) 2014;6:143-152.

12 Pagès F, Galon J, Dieu-Nosjean MC, et al.: Immune infiltration in human tumors: a prognostic factor that should not be ignored. Oncogene 2010;29:1093-1102.

13 Calabrò A, Beissbarth T, Kuner R, et al.: Effects of infiltrating lymphocytes and estrogen receptor on gene expression and prognosis in breast cancer. Breast Cancer Res Treat 2009;116:69-77.

14 Rody A, Holtrich U, Pusztai L, et al.: T-cell metagene predicts a favorable prognosis in estrogen receptornegative and HER2-positive breast cancers. Breast Cancer Res 2009;11:R15.

15 Ono M, Tsuda H, Shimizu C, et al.: Tumor-infiltrating lymphocytes are correlated with response to neoadjuvant chemotherapy in triple-negative breast cancer. Breast Cancer Res Treat 2012;132:793-805.

16 Lee HJ, Seo JY, Ahn JH, et al.: Tumor-associated lymphocytes predict response to neoadjuvant chemotherapy in breast cancer patients. J Breast Cancer 2013; 16:32-39.

17 Denkert C, Loibl S, Noske A, et al.: Tumor-associated lymphocytes as an independent predictor of response to neoadjuvant chemotherapy in breast cancer. J Clin Oncol 2010;28:105-113.

18 West NR, Milne K,Truong PT, et al.: Tumor-infiltrating lymphocytes predict response to anthracyclinebased chemotherapy in estrogen receptor-negative breast cancer. Breast Cancer Res 2011;13:R126

19 Loi S, Sirtaine N, Piette F, et al.: Prognostic and predictive value of tumor-infiltrating lymphocytes in a phase III randomized adjuvant breast cancer trial in nodepositive breast cancer comparing the addition of docetaxel to doxorubicin with doxorubicin-based chemotherapy: BIG 02-98. J Clin Oncol 2013;31:860-867.
20 Andre F, Dieci MV, Dubsky P, et al.: Molecular pathways: involvement of immune pathways in the therapeutic response and outcome in breast cancer. Clin Cancer Res 2013;19:28-33.

21 Loi S: Tumor-infiltrating lymphocytes, breast cancer subtypes and therapeutic efficacy. Oncoimmunology 2013;2:e24720

22 Feuerer M, Beckhove P, Garbi N, et al.: Bone marrow as a priming site for $\mathrm{T}$-cell responses to blood-borne antigen. Nature Med 2003;9:1151-1157.

23 Stefanovic S, Schuetz F, Sohn C, et al.: Bone marrow microenvironment in cancer patients: immunological aspects and clinical implications. Cancer Metastasis Rev 2013;32:163-178

24 Feuerer M, Beckhove P, Mahnke Y, et al.: Bone marrow microenvironment facilitating dendritic cell: $\mathrm{CD} 4$ $\mathrm{T}$ cell interactions and maintenance of CD4 memory. Int J Oncol 2004;25:867-876.

25 Mazo IB, Honczarenko M, Leung H, et al.: Bone marrow is a major reservoir and site of recruitment for central memory CD8+ T cells. Immunity 2005;22:259-270.

26 Schirrmacher V, Feuerer M, Fournier P, et al.: T-cell priming in bone marrow: the potential for long-lasting protective anti-tumor immunity. Trends Mol Med 2003;9:526-534.

27 Khazaie K, Prifti S, Beckhove P, et al.: Persistence of dormant tumor cells in the bone marrow of tumor cell-vaccinated mice correlates with long-term immunological protection. Proc Natl Acad Sci USA 1994;91: 7430-7434.

28 Schirrmacher V, Feuerer M, Beckhove P, et al.: T cell memory, anergy and immunotherapy in breast cancer. J Mammary Gland Biol Neoplasia 2002;7:201-208.

29 Bai L, Beckhove P, Feuerer M, et al.: Cognate interactions between memory $\mathrm{T}$ cells and tumor antigen-presenting dendritic cells from bone marrow of breast cancer patients: bidirectional cell stimulation, survival and antitumor activity in vivo. Int J Cancer 2003;103:73-83. 
30 Mahnke YD, Schwendemann J, Beckhove P, Schirrmacher V: Maintenance of long-term tumour-specific T-cell memory by residual dormant tumour cells. Immunology 2005;115:325-336.

-31 Solomayer EF, Feuerer M, Bai L, et al.: Influence of adjuvant hormone therapy and chemotherapy on the immune system analysed in the bone marrow of patients with breast cancer. Clin Cancer Res 2003;9:174-180.

32 Zinkernagel RM, Bachmann MF, Kündig TM, et al.: On immunological memory. Ann Rev Immunol 1996; 14:333-367.

33 Goldrath AW, Bevan MJ: Selecting and maintaining a diverse T-cell repertoire. Nature 1999;402:255-262.

34 Lanzavecchia A, Sallusto F: From synapses to immunological memory: the role of sustained T cell stimulation. Curr Opin Immunol 2000;12:92-98.

-35 Schuetz F, Ehlert K, Ge Y, et al.: Treatment of advanced metastasized breast cancer with bone marrow-derived tumour-reactive memory T cells: a pilot clinical study. Cancer Immunol Immunother 2009;58:887-900.

36 Domschke C, Ge Y, Bernhardt I, et al.: Long-term survival after adoptive bone marrow $\mathrm{T}$ cell therapy of advanced metastasized breast cancer: follow-up analysis of a clinical pilot trial. Cancer Immunol Immunother 2013;62:1053-1060.

37 Vesely MD, Kershaw MH, Schreiber RD, Smyth MJ: Natural innate and adaptive immunity to cancer. Ann Rev Immunol 2011;29:235-271.

38 Zhou G, Levitsky H: Towards curative cancer immunotherapy: overcoming posttherapy tumor escape. Clin Dev Immunol 2012;2012:124187.

- 39 Litzinger MT, Fernando R, Curiel TJ, et al.: IL-2 immunotoxin denileukin diftitox reduces regulatory $\mathrm{T}$ cells and enhances vaccine-mediated T-cell immunity. Blood 2007;110:3192-3201.

40 Ruter J, Barnett BG, Kryczek I, et al.: Altering regulatory $\mathrm{T}$ cell function in cancer immunotherapy: a novel means to boost the efficacy of cancer vaccines. Front Biosc 2009;14:1761-1770.

41 Salagianni M, Lekka E, Moustaki A, et al.: NK cell adoptive transfer combined with Ontak-mediated regulatory $\mathrm{T}$ cell elimination induces effective adaptive antitumor immune responses. J Immunol 2011;186: 3327-3335.

42 Beyer M, Schultze JL: Regulatory T cells in cancer. Blood 2006;108:804-811.

43 Petrausch U, Poehlein CH, Jensen SM, et al.: Cancer immunotherapy: the role regulatory $\mathrm{T}$ cells play and what can be done to overcome their inhibitory effects. Curr Mol Med 2009;9:673-682.

44 Lutsiak MEC, Semnani RT, De Pascalis R, et al.: Inhibition of $\mathrm{CD} 4(+) 25+\mathrm{T}$ regulatory cell function implicated in enhanced immune response by low-dose cyclophosphamide. Blood 2005;105:2862-2868.

45 Ghiringhelli F, Menard C, Puig PE, et al.: Metronomic cyclophosphamide regimen selectively depletes $\mathrm{CD} 4+\mathrm{CD} 25+$ regulatory $\mathrm{T}$ cells and restores $\mathrm{T}$ and $\mathrm{NK}$ effector functions in end stage cancer patients. Cancer Immunol Immunother 2007;56:641-648.

46 Ge Y, Domschke C, Stoiber N, et al.: Metronomic cyclophosphamide treatment in metastasized breast cancer patients: immunological effects and clinical outcome. Cancer Immunol Immunother 2012;61:353-362.

47 Disis ML, Bernhard H, Jaffee EM: Use of tumourresponsive $\mathrm{T}$ cells as cancer treatment. Lancet 2009; 373:673-683.

48 Yee C, Thompson JA, Roche P, et al.: Melanocyte destruction after antigen-specific immunotherapy of melanoma: direct evidence of $\mathrm{t}$ cell-mediated vitiligo. J Exp Med 2000;192:1637-1644.
49 Dudley ME, Wunderlich JR, Yang JC, et al.: A phase I study of nonmyeloablative chemotherapy and adoptive transfer of autologous tumor antigen-specific T lymphocytes in patients with metastatic melanoma. J Immunother 2002;25:243-251.

50 Kunert A, Straetemans T, Govers C, et al.: TCR-engineered $\mathrm{T}$ cells meet new challenges to treat solid tumors: choice of antigen, $\mathrm{T}$ cell fitness, and sensitization of tumor milieu. Front Immunol 2013;4:363.

51 Curigliano G, Viale G, Ghioni M, et al.: Cancer-testis antigen expression in triple-negative breast cancer. Ann Oncol 2011;22:98-103.

52 Ayyoub M, Scarlata CM, Hamai A, et al.: Expression of MAGE-A3/6 in primary breast cancer is associated with hormone receptor negative status, high histologic grade, and poor survival. J Immunother 2014;37:73-76.

53 Katz T, Avivi I, Benyamini N, et al.: Dendritic cell cancer vaccines: from the bench to the bedside. Rambam Maimonides Med J 2014; 5:e0024.

54 Ravelli A, Reuben JM, Lanza F, et al.: Immune-related strategies driving immunotherapy in breast cancer treatment: a real clinical opportunity. Expert Rev Anticancer Ther 2015;15:689-702.

55 Feuerer M, Beckhove P, Bai L, et al.: Therapy of human tumors in NOD/SCID mice with patient-derived reactivated memory $\mathrm{T}$ cells from bone marrow. Nature Med 2001;7:452-458.

56 Beckhove P, Feuerer M, Dolenc M, et al.: Specifically activated memory $\mathrm{T}$ cell subsets from cancer patients recognize and reject xenotransplanted autologous tumors. J Clin Invest 2004;114:67-76.

57 Stefanovic S, Schuetz F, Sohn C, et al.: Adoptive immunotherapy of metastatic breast cancer: present and future. Cancer Metastasis Rev 2014;33:309-320.

58 Chirgwin JM, Guise TA: Molecular mechanisms of tumor-bone interactions in osteolytic metastases. Crit Rev Eukaryot Gene Expr 2000;10:159-178.

59 Domschke C, Schuetz F, Ge Y, et al.: Intratumoral cytokines and tumor cell biology determine spontaneous breast cancer-specific immune responses and their correlation to prognosis. Cancer Res 2009;69:8420-8428.

$60 \mathrm{Li}$ MO, Wan YY, Sanjabi S, et al.: Transforming growth factor-beta regulation of immune responses. Ann Rev Immunol 2006;24:99-146.

61 Chen W, Jin W, Hardegen N, et al.: Conversion of peripheral CD4+ CD25- naive $\mathrm{T}$ cells to $\mathrm{CD} 4+\mathrm{CD} 25+$ regulatory $\mathrm{T}$ cells by TGF-beta induction of transcription factor Foxp3. J Exp Med 2003;198:1875-1886.

62 Oelke M, Maus MV, Didiano D, et al.: Ex vivo induction and expansion of antigen-specific cytotoxic T cells by HLA-Ig-coated artificial antigen-presenting cells. Nature Med 2003;9:619-624.

63 Chmielewski M, Abken H: CAR T cells transform to trucks: chimeric antigen receptor-redirected $\mathrm{T}$ cells engineered to deliver inducible IL-12 modulate the tumour stroma to combat cancer. Cancer Immunol Immunother 2012;61:1269-1277.

64 Koehler P, Schmidt P, Hombach A, et al.: Engineered $\mathrm{T}$ cells for the adoptive therapy of B-cell chronic lymphocytic leukaemia. Adv Hematol 2012;2012:595060.

$65 \mathrm{Wu}$ R, Forget MA, Chacon J, et al.: Adoptive T-cell therapy using autologous tumor-infiltrating lymphocytes for metastatic melanoma: current status and future outlook. Cancer J 2012;18:160-175.

66 Ellebaek E, Iversen TZ, Junker N, et al.: Adoptive cell therapy with autologous tumor infiltrating lymphocytes and low-dose interleukin-2 in metastatic melanoma patients. J Transl Med 2012;10:169.
7 Radvanyi LG, Bernatchez C, Zhang M, et al.: Specific lymphocyte subsets predict response to adoptive cell therapy using expanded autologous tumor-infiltrating lymphocytes in metastatic melanoma patients. Clin Cancer Res 2012;18:6758-6770.

68 Besser MJ, Shapira-Frommer R, Treves AJ, et al.: Clinical responses in a phase II study using adoptive transfer of short-term cultured tumor infiltration lymphocytes in metastatic melanoma patients. Clin Cancer Res 2010;16:2646-2655.

69 West NR, Kost SE, Martin SD, et al.: Tumour-infiltrating FOXP3(+) lymphocytes are associated with cytotoxic immune responses and good clinical outcome in oestrogen receptor-negative breast cancer. $\mathrm{Br} \mathrm{J}$ Cancer 2013;108:155-162.

70 Shi H, Liu L, Wang Z: Improving the efficacy and safety of engineered $T$ cell therapy for cancer. Cancer Lett 2012;328:191-197.

71 Cordova A, Toia F, La Mendola C, et al.: Characterization of human $\gamma \delta$ T lymphocytes infiltrating primary malignant melanomas. PloS One 2012;7:e49878.

72 Hadrup SR: The antigen specific composition of melanoma tumor infiltrating lymphocytes? Oncoimmunology 2012;1:935-936.

73 Morgan RA, Dudley ME, Wunderlich JR, et al.: Cancer regression in patients after transfer of genetically engineered lymphocytes. Science 2006;314:126-129.

74 Johnson LA, Morgan RA, Dudley ME, et al.: Gene therapy with human and mouse T-cell receptors mediates cancer regression and targets normal tissues expressing cognate antigen. Blood 2009;114:535-546.

75 Robbins PF, Morgan RA, Feldman SA, et al.: Tumor regression in patients with metastatic synovial cell sarcoma and melanoma using genetically engineered lymphocytes reactive with NY-ESO-1. J Clin Oncol 2011;29:917-924.

76 Yvon E, Del Vecchio M, Savoldo B, et al.: Immunotherapy of metastatic melanoma using genetically engineered GD2-specific T cells. Clin Cancer Res 2009;15: 5852-5860.

77 Lo ASY, Ma Q, Liu DL, et al.: Anti-GD3 chimeric sFv$\mathrm{CD} 28 / \mathrm{T}$-cell receptor zeta designer $\mathrm{T}$ cells for treatment of metastatic melanoma and other neuroectodermal tumors. Clin Cancer Res 2010;16:2769-2780.

78 Burns WR, Zhao Y, Frankel TL, et al.: A high molecular weight melanoma-associated antigen-specific chimeric antigen receptor redirects lymphocytes to target human melanomas. Cancer Res 2010;70:3027-3033.

79 Sage PT, Francisco LM, Carman CV, et al.: The receptor PD-1 controls follicular regulatory T cells in the lymph nodes and blood. Nat Immunol 2013;14:152-161.

80 Wimberly H, Brown JR, Schalper K, et al.: PD-L1 expression correlates with tumor-infiltrating lymphocytes and response to neoadjuvant chemotherapy in breast cancer. Cancer Immunol Res 2015;3:326-332.

81 Nanda R, Chow LQ, Dees EC, et al. A phase Ib study of pembrolizumab (MK-3475) in patients with advanced triple-negative breast cancer. SABCS 2014; abstr S1-09.

82 Topalian SL, Sznol M, McDermott, et al.: Survival, durable tumor remission, and long-term safety in patients with advanced melanoma receiving nivolumab. J Clin Oncol 2014;32:1020-1030.

83 Postow MA, Chesney J, Pavlick AC, et al.: Nivolumab and ipilimumab versus ipilimumab in untreated melanoma. N Engl J Med 2015;372:2006-2017. 